\title{
Philosophiques
}

\section{Amartya Sen, Éthique et économique (et autres essais); traduit de l'anglais par Sophie Marnat, Paris, Presses universitaires de France (collection « Philosophie morale »), 1993, 364 pages.}

\section{J. Nicolas Kaufmann}

Volume 22, numéro 1, printemps 1995

URI : https://id.erudit.org/iderudit/027319ar

DOI : https://doi.org/10.7202/027319ar

Aller au sommaire du numéro

Éditeur(s)

Société de philosophie du Québec

ISSN

0316-2923 (imprimé)

1492-1391 (numérique)

Découvrir la revue

Citer ce compte rendu

Kaufmann, J. N. (1995). Compte rendu de [Amartya Sen, Éthique et économique (et autres essais); traduit de l'anglais par Sophie Marnat, Paris, Presses universitaires de France (collection " Philosophie morale »), 1993, 364 pages.] Philosophiques, 22(1), 173-176. https://doi.org/10.7202/027319ar d'utilisation que vous pouvez consulter en ligne.

https://apropos.erudit.org/fr/usagers/politique-dutilisation/ 
Amartya Sen, Éthique et économique (et autres essais); traduit de l'anglais par Sophie Marnat, Paris, Presses universitaires de France (collection « Philosophie morale »), 1993, 364 pages.

\section{par J. Nicolas Kaufmann}

Le corpus du livre est formé d'une version révisée des trois conférences que Sen a prononcées en 1986 à l'Université de Californie dans le cadre des « Royer Lectures ». Sept autres essais qui ont été publiés entre 1981 et 1991 s'y trouvent ajoutés pour constituer un ensemble de textes qui se complètent mutuellement et constituent un tout très cohérent, une série de réflexions sur l'interfécondation de l'éthique et de l'économie.

Rappelant ce qui fut au cœur de l'éthique d'Aristote concernant « la vie bonne »- agir en vertu de ce que nous percevons comme restrictions impartiales à imposer à la poursuite des intérêts égoïstes dans le but d'accomplir les fins de la communauté, projet auquel se subordonnerait l'économie -, Sen déplore le fait que l'économie moderne se soit éloignée de ce projet éthique pour uniquement se soucier, dans une perspective instrumentale, des moyens à mettre en œuvre pour réaliser des fins données par ailleurs. Cette séparation de plus en plus marquée entre l'éthique et l'économie constituerait une perte 
non seulement pour l'économie, mais serait faite également au détriment de l'éthique. Sen consacre les trois conférences à l'examen de la portée de cette perte. Dans le premier essai ( comportement économique et sentiments moraux »), il situe clairement l'enjeu de sa critique qui concerne le rétrécissement que devait subir l'analyse économique à partir du moment où, aussi bien la théorie du choix du consommateur (d'Adam Smith à Garry Becker) que les différentes théories économiques du bien-être (de Pareto, Pigou à PhelpsBrown), trouvaient leurs assises dans une donnée ultime qui, dans la tradition de Hobbes, constituait la seule motivation importante de l'agir humain en général et des choix économiques en particulier : les intérêts égoïstes. Sen s'applique à démontrer que l'usage qui est ainsi fait de la notion (utilitariste) d'utilité est conceptuellement défectueux, descriptivement inadéquat et normativement insoutenable (comme Smith, d'ailleurs, l'avait déjà entrevu). Il s'agit d'une conception trop étroite qui ne pouvait conduire qu'à des doctrines simplistes.

Sen prolonge sur le terrain de l'économie du bien-être la critique esquissée, en indiquant dans la seconde conférence (essai 2 : « Jugements sur l'économie et philosophie morale ») que le critère de Pareto-optimalité constituait d'un point de vue éthique un critère extrêmement étroit pour évaluer les réalisations sociales du fait que ce critère était limité aux utilités individuelles et ne pouvait inclure les utilités associées à des distributions mêmes des utilités personnelles. Il remarque, avec pertinence, que la littérature welfariste était grevée du problème d'interprétation de la notion (technique) d'utilité assimilée à « avantage », « bonheur », « bien-être », « satisfaction de besoin ou de désir » et à d'autres notions apparentées. Il identifie deux autres limites du welfarisme : absence de différenciation entre bien-être et accomplissement dans l'action, absence d'utilité pour la liberté. C'est dans la troisième conférence (essai 3 : « Liberté et conséquences ») que Sen propose plus constructivement de repousser ces limites tout en poursuivant sa critique de la conception utilitariste de la personne. En introduisant plusieurs distinctions concernant le concept de bien-être, en termes d'accomplissement, de liberté (champ de possibles) et de qualité d'agent (combinaison d'accomplissement et de liberté), il montre clairement que les quatre sortes d'information requises pour caractériser le bien-être d'une personne ne se laissent pas homogénéiser par la notion étroite d'utilité. Il trace ainsi la voie, à partir de la perspective éthique, pour l'enrichissement de l'analyse économique du bien-être. Cependant, Sen devait constater et prouver à ce sujet un théorème en théorie du choix social, l'incompatibilité entre les conditions de Paretooptimalité (P), de non-restriction du domaine de choix (U) et de liberté (L) qui exige qu'au moins dans un secteur les préférences individuelles priment sur toutes les autres ( $c f$. essai $6: 1983$, « Liberté et choix social »). Le recours à la liberté permet également à Sen de faire une lecture critique de la théorie de la 
justice de Rawls en matière de choix social. A cet effet, Sen argumente (dans l'essai 8 : I99o, « L'évaluation de la justice doit-elle se fonder sur les moyens ou sur les libertés? ») en faveur d'une double base pour asseoir notre jugement en matière de justice : les moyens dont peuvent disposer les individus, d'une part, et les variations qui existent entre des individus dans la capacité de convertir ces moyens en liberté d'accomplir des actions, d'autre part.

Le caractère descriptivement inadéquat de l'homo aconomicus agissant uniquement en fonction de ses préférences égoïstes avait depuis longtemps été mis en évidence par Sen, notamment dans son célèbre texte de l'essai 4 (1983, «Des idiots rationnels 》). Sen y met en évidence deux formes de comportement, la compassion (« penser au sous-développement me rend malade ») et l'engagement («penser au sous-développement me dispose à faire quelque chose ») dont le dernier a de multiples implications économiques. principalement dans le domaine de la consommation des biens publics (voir le paradoxe d'Olson). L'analyse de ces comportements permet à Sen de conclure que la structure de la théorie traditionnelle basée sur les préférences (individuelles) égoïstes est trop rudimentaire, qu'à côté des préférences naturelles il faudrait faire place à des méta-préférences (voir la conférence séminale de Bristol, 1974) pouvant être interprétées en termes de «préférences morales », « priorités sociales », « intérêts de classe », etc.

Sen étend sa critique des lacunes de l'utilitarisme classique en comparant deux conceptions du droit (essai 5 : 1982, « Les droits et la question de l'agent 》), une conception utilitariste développée dans la perspective conséquentialiste issue de la théorie du bien-être (Hart, Parfit, Hare) et fondée sur le critère de la somme totale des utilités individuelles, et une conception déontologique fondée sur des contraintes qui ont une importance intrinsèque et qui ne doivent pas être violées mème si cela améliorerait la situation en termes d'utilité. Sen illustre les lacunes respectives de ces doctrines par l'examen d'un exemple des interdépendances entre agents multiples du genre: la seule façon d'éviter que A soit violé par B pourrait être que $\mathrm{C}$ arrive sur les lieux dans une voiture dérobée à $\mathrm{D}$. La discussion instructive de Sen permet de dépasser ces lacunes par le recours à un système des droits-buts conçu comme un système moral « dans lequel le respect et la violation des droits sont compris parmi les buts, intégrés à l'évaluation des situations puis appliqués aux choix des actions par des liens de conséquences » (130). Sen reconnaît donc la supériorité du point de vue utilitariste conséquentialiste, à condition qu'il soit élargi en prenant en considération les pouvoirs d'agir qui fondent un système de droits à des capabilités, notion que dépasse celle des droits se rapportant aux libertés dites positives et celle des droits liés aux libertés négatives.

Un dernier volet de la critique des fondements de l'utilitarisme comme doctrine morale appliquée à l'économie du bien-ètre est déployé par Sen dans 
l'essai 7 (1982, «Quelle égalité ?») en comparant différentes notions d'égalité (et de justice comme équité) : égalité utilitariste, égalité d'utilité totale (leximin), égalité selon Rawls (principe de différence et maximin). Cest par une argumentation serrée concernant les implications qu'ont les trois variantes pour le cas des handicapés que Sen met en évidence les limites préoccupantes de ces doctrines et plaide en faveur de sa propre conception basée sur les capabilités (voir les essais 2 et 5 ). C'est la possibilité d'exercer les capacités d'agent, et non l'utilité marginale individuelle, le total des utilités individuelles ou les biens sociaux premiers, qui doit être prise pour la mesure de base pour établir des inégalités et apprécier les mesures correctives. Ce point de vue est illustré à l'aide d'une analyse de deux situations concrètes, la discrimination sexuelle (essai 9 : 1990, «La distinction entre les sexes et les conflits de coopération »), la répartition du revenu (essai ro, 198I, « Problèmes éthiques dans la répartition du revenu »).

La discussion que Sen entretient à travers les essais publiés dans ce livre est toujours extrêmement bien informée et documentée par des références à une littérature abondante (47 pages d'entrées bibliographiques) qu'il suppose connue. Quant aux rapports entre économie (du bien-être) et éthique, fil conducteur des textes colligés, Sen nous laisse, bien malgré lui, l'impression d'un déséquilibre : l'économie du bien-être est l'équivalent économique d'un « trou noir »; beaucoup de choses peuvent y entrer, mais rien n'en ressort.

La traduction des textes est très soignée, comme l'illustre le glossaire $\left(357^{-}\right.$ $360)$ que le directeur de la collection a bien voulu incorporer au volume.

\author{
Département de philosophie \\ Université du Québec à Trois-Rivières
}

